\title{
IR 820 dye encapsulated in polycaprolactone glycol chitosan: Poloxamer blend nanoparticles for photo immunotherapy for breast cancer
}

\author{
Piyush Kumar, Rohit Srivastava * \\ Department of Biosciences and Bioengineering, Indian Institute of Technology-Bombay, Powai, Mumbai 400076, India
}

\section{A R T I C L E I N F O}

\section{Article history:}

Received 13 April 2015

Received in revised form 20 July 2015

Accepted 4 August 2015

Available online 7 August 2015

\section{Keywords:}

IR 820

Photothermal therapy

TNF- $\alpha$

Poloxamer

Biocompatibility

Reactive oxygen species

\begin{abstract}
A B S T R A C T
In the present study, we have fabricated biocompatible and biodegradable monodisperse IR 820 encapsulated polycaprolactone (PCL) glycol chitosan (GC): Poloxamer blend nanoparticles (PP-IR NPs) for imaging and effective photo-immunotherapy. IR 820 has been used as an imaging and photothermal agent whereas glycol chitosan (GC) as an immunostimulatory agent. The combination of IR 820, poloxamer, and GC can be used effectively for photoimmunotherapy for cancer, drug-resistant and TNF- $\alpha$ resistant estrogen positive breast cancer. PP-IR NPs are stable in aqueous solution. The uniform size of $100-220 \mathrm{~nm}$ with a high zeta value of $+38 \pm 2 \mathrm{mV}$ led them to accumulate in cancer cells. Laser treatment did not affect the morphology of PP-IR NPs as observed under the transmission electron microscope (TEM). In vitro cytotoxicity studies on MCF-7 cells showed enhanced toxicity upon laser treatment. Further, we validated the cell death by reactive oxygen species (ROS) production. Our studies thus showed that PP-IR NPs are effective in suppressing metastatic cancer as the combinational therapy leads to the formation of apoptotic bodies in MCF-7 cells.
\end{abstract}

(c) 2015 Elsevier B.V. All rights reserved.

\section{Introduction}

Polymeric nanosystem either natural or synthetic has gained widespread popularity in the field of drug delivery and tissue engineering. Small size, ease of shaping and ability to deliver a wide range of drugs to specific sites have made them a potential candidate for drug delivery system [1]. Natural polymers like proteins and polysaccharides have not been widely used owing to certain limitations such as purity and requirement to cross link to the encapsulated drug. Owing to rectify these limitations, synthetic polymer came into existence. The widely used synthetic polymers are polylactic acid (PLA), polyglycolide (PGA), poly(lactic-co-glycolic acid) PLGA, and polycaprolactone (PCL) [2]. Drug loaded PLGA nanoparticles have been widely used to its nature to encapsulate both hydrophobic and hydrophilic drugs. However, the high cost of PLGA throws light on a cheaper alternative like PCL for a new carrier. The low melting point $\left(59-64{ }^{\circ} \mathrm{C}\right)$, optimum blending compatibility, cost effectiveness and biocompatibility have made PCL a potential carrier in controlled drug delivery, especially in cancer therapeutics [3]. The therapeutic activity and stability of the drug-polymer conjugate are much higher in PCL compared to PLGA [4].

Poloxamer is a non-ionic, non-toxic, tri-block copolymer surfactant widely used in clinical trials and pharmaceutical formulations [5-7]. The addition of poloxamer has been shown to reduce the size of nanoparticles (NPs) and enhance the release of the drugs from PCL matrix.

\footnotetext{
* Corresponding author at: NanoBios Lab, Department of Biosciences and Bioengineering, Indian Institute of Technology-Bombay, Powai, Mumbai 400076, India.

E-mail address: rsrivasta@iitb.ac.in (R. Srivastava).
}

Poloxamer has also been shown to increase cellular uptake of docetaxel loaded PCL-poloxamer (di-block polymeric) NPs in docetaxel resistant MCF-7-TAX 30 human breast cancer cells in comparison to docetaxel loaded PCL NPs alone [8]. A similar study has been reported with docetaxel or paclitaxel loaded PLGA and poloxamer blend NPs wherein drug loaded PLGA poloxamer blend NPs showed potent efficacy in drugresistant human breast cancer cells due to increased uptake and enhanced release $[6,9]$. Poloxamer has been reported to enhance the transfection efficiency by minimizing the cell injury due to electric shock [10, 11]. Amphiphilic GC, a water-soluble chitosan derivative at physiological $\mathrm{pH}$ 7.4, has been widely used in the formulation of anti-cancer drugs, nucleic acid transfection and imaging agents for diagnostic purposes [12-18].

Tumor re-occurrence and drug resistance are the main limitations of conventional cancer therapy. To increase the efficacy and to combat these limitations, the combinational therapy of chemotherapy and radiation therapy is the need of hour. However, harmful exposure of radiation to healthy cells and recurrent tumors has been emphasized on the safer alternative like laser chemotherapy or photothermal therapy [19]. The photothermal therapy uses a light source or laser in the nearinfrared region (NIR) that penetrates deep into the tissue. To get thermal ablation in the deep seated tumor in tissues, it is worth to use a light absorbing agent that can convert electromagnetic radiation into heat energy such as metals and dyes. Metallic materials like gold can easily be tuned in NIR range. However, their clearance through the body is still the debated issue. On the other hand, organic dyes such as Indocyanine dyes have emerged as new cancer theranostic agent owing to its biocompatible and biodegradable nature. 
Indocyanine dyes having an absorbance in NIR range around $800 \mathrm{~nm}$ have been widely used as photothermal agents. The most commonly used Indocyanine dye is the Indocyanine Green (ICG) which has been explored for multimodal imaging and photothermal agent [20-23]. The poor aqueous solubility, short half-life and photobleaching properties of ICG limit its use as a good photothermal agent. In addition, ICG has been modified to increase stability and efficacy without affecting much of its photothermal efficiency. This modified ICG is termed as IR 820 which is a cheaper alternative to ICG. The presence of chlorobenzene ring in IR 820 makes it more stable than ICG. The estimated halflife for clearance of IR 820 in the body is around $34 \mathrm{~h}$ which is much longer than that of ICG (150-180 s) [24]. IR 820 has been widely explored as bioimaging agents in mouse [25]. The ease of conjugation and good stability in blood circulation make it an ideal choice for multimodal imaging and therapy [26-28]. In the previous studies, we have focused only on IR 820 dye based photothermal therapy for cancer treatment [29]. This can be further improved by adding immune adjuvant like GC for an immunological response. In the current study, we have focused on the significance of each component and their role in cancer treatment. PCL has been chosen for drug loading, poloxamer as an apoptosis stimulator and GC as immune adjuvant for immune response. We developed PP-IR NPs for effective photo-immunotherapy for efficient cancer therapy.

In the present study, we synthesized biocompatible, biodegradable and uniform PP-IR NPs. We performed biocompatibility test in NIH3T3 cells, cellular uptake in vitro, photothermal efficiency and heat generation property of these NPs on human estrogen positive breast cancer (MCF-7) cells. We have also studied the cell death by fluorescence microscopy and reactive oxygen species (ROS). We explored the synergistic effect of IR 820, GC and poloxamer on MCF-7 cells. The rationale of this paper was to focus on photo-immunotherapy to target multiple drugs resistant (MDR) and hyperthermia resistant cancers.

\section{Experimental}

\subsection{Materials}

Polycaprolactone, poly(vinyl alcohol) 13-23 K and glycol chitosan (MW: 100,000-300,000, the degree of polymerization $\geq 400$, degree of purity $\geq 75-85 \%$ ) were purchased from Sigma-Aldrich. Ltd. L-Ascorbic acid was purchased from SRL India. Poloxamer 188 was purchased from SDSF Germany. MCF-7 cells were procured from NCCS Pune. All other chemicals and reagents used were of analytical grade.

\subsection{Formulation of PP-IR NPS}

PP-IR NPs were prepared using a modified solvent evaporation method [30]. Briefly PCL and poloxamer (9:1) were dissolved in dichloromethane (DCM). IR 820 was dissolved in water and added to the organic phase to make the primary emulsion and sonicated for $1 \mathrm{~min}$. The primary emulsion was slowly added to the aqueous phase containing GC $(1 \mathrm{mg} / \mathrm{mL})$ to form the secondary emulsion. The emulsion was sonicated for $5 \mathrm{~min}$ at $50 \%$ amplitude. The NP suspension was allowed to evaporate at room temperature. Finally, NPs were obtained by centrifuging the suspension at $14,000 \times \mathrm{g}$ for $20 \mathrm{~min}$ followed by three washes with water.

Fluorescein isothiocyanate dextran (FITC-D) loaded NPs for cellular uptake studies were fabricated by adding FITC-D in water to the organic phase containing polymers. The rest of the procedure was similar to the above-mentioned NP formulations.

\subsection{Characterization}

The size and distribution of particles formed were analyzed by Dynamic Light Scattering (DLS, Model BI 200 SM Brookhaven Instruments, USA). Similarly, surface charge was analyzed by Zeta plus (Brookhaven
Instruments, USA). The NPs were further examined by Field Emission Gun-Scanning Electron Microscope (FEG-SEM, JEOL JSM 7600F) and transmission electron microscope (TEM, PHILIPS CM 200) for morphological evaluation, size and aggregation.

\subsection{Degradation study of PCL polyglycol chitosan IR 820 nanoparticle (PPIR) effect}

Nearly $200 \mu \mathrm{L}$ of samples was subjected to different conditions such as visible daylight and $\mathrm{UV}(375 \mathrm{~nm})$ at a temperature of $37^{\circ} \mathrm{C}$ for $30 \mathrm{~min}$ and with laser for $5 \mathrm{~min}$ at a distance of $1 \mathrm{~cm}$ pathlength. Treated samples were analyzed by TEM for any morphological changes.

\subsection{Photothermal transduction effect}

Photothermal effect of nanoparticles was studied using an $808 \mathrm{~nm}$ infrared laser $(0.5 \mathrm{~W})$ along with a digital thermocouple thermometer (Okaton Mini infra Pro-6; accuracy $\pm 1.5 \%$ reading value). Briefly, $200 \mu \mathrm{L}(200 \mu \mathrm{g} / \mathrm{mL})$ suspensions of NPs were irradiated by an $808 \mathrm{~nm}$ laser in a 96 well plate in the water bath. The rise in temperature was measured at a gradual interval of $1 \mathrm{~min}$ to $5 \mathrm{~min}$. All the experiments were conducted at $37^{\circ} \mathrm{C}$.

\subsection{Cellular uptake studies}

MCF-7 cells $\left(1 * 10^{5}\right)$ were seeded in 12 well plates maintained at $5 \%$ $\mathrm{CO}_{2}$ at $37^{\circ} \mathrm{C}$. After $24 \mathrm{~h}$, cells were washed with phosphate buffer saline (PBS) pH 7.4 to remove unattached cells. The cells were further incubated with media containing FITC-D loaded NPs for $4 \mathrm{~h}$. The cells were washed thrice with PBS to remove free NPs. Cells were fixed in 3.7\% formaldehyde for 15 min at room temperature and washed thrice with PBS. The fixed cells were observed under the confocal laser scanning microscope (Olympus) at $\lambda_{\mathrm{ex}} 488 \mathrm{~nm}$ and $\lambda_{\mathrm{em}} 520 \mathrm{~nm}$.

\subsection{Biocompatibility study}

NIH3T3 $\left(2 * 10^{4}\right)$ cells were seeded in 96 well plates and incubated for $24 \mathrm{~h}$ under $5 \% \mathrm{CO}_{2}$ at $37{ }^{\circ} \mathrm{C}$. The medium was replaced with fresh complete media containing NPs from $100-1000 \mu \mathrm{g} / \mathrm{mL}$. The plate was further incubated for $24 \mathrm{~h}$ followed by cell viability analysis using MTT assay. Briefly, samples were incubated with MTT dye $(500 \mu \mathrm{g} / \mathrm{ml})$ for $4 \mathrm{~h}$ till there was a formation of formazan crystals. They were carefully dissolved in $150 \mu \mathrm{L}$ of DMSO and read on a microplate reader (Molecular devices, USA) at a wavelength of $560 \mathrm{~nm}$ with reference at $690 \mathrm{~nm}$.

\subsection{Photothermal therapy}

For photothermal effect, MCF-7 $\left(1 * 10^{4}\right)$ cells were seeded into 96 well plates and incubated for $24 \mathrm{~h}$ under $5 \% \mathrm{CO}_{2}$ at $37^{\circ} \mathrm{C}$. The medium was replaced with fresh media with $10 \%$ serum containing $200 \mu \mathrm{g}$ of NPs and incubated for $4 \mathrm{~h}$. Later, cells were washed with PBS and replaced with fresh complete media. Prior to laser application, the plate was incubated at $37{ }^{\circ} \mathrm{C}$ in a dry bath incubator and cells were irradiated with a $0.5 \mathrm{~W} 808 \mathrm{~nm}$ laser for $5 \mathrm{~min}$. To determine cytotoxic effect cells were further subjected to MTT assay.

\subsection{Cell death study by Hoechst PI double staining}

For live/dead, apoptotic and necrotic cell visualization, MCF 7 $\left(0.5 * 10^{5}\right)$ cells were seeded on the cover slip in 12 well plates under $5 \% \mathrm{CO}_{2}$ at $37^{\circ} \mathrm{C}$. The remaining protocol was similar to photothermal effect as described earlier. At the end of laser application, cells were stained with Hoechst $33342(10 \mu \mathrm{g} / \mathrm{mL})$ and incubated at $37{ }^{\circ} \mathrm{C}$ for 20 min in PBS containing $\mathrm{Mg}^{++}$and $\mathrm{Ca}^{++}$. Cells were washed with PBS followed by Propidium Iodide (PI, $10 \mu \mathrm{g} / \mathrm{mL}$ ) staining for $5 \mathrm{~min}$. 
The cells were washed thrice with PBS and visualized under a Leica fluorescent microscope (Germany).

\subsection{Reactive oxygen species production}

ROS production was studied as per standard protocol [31]. Briefly NPs were added $4 \mathrm{~h}$ prior to laser treatment. $2 \mathrm{~h}$ prior to laser treatment, $1 \mu \mathrm{g} / \mathrm{mL}$ ascorbic acid was added to quench internal ROS production. Further, the rest of procedure was similar to photothermal therapy on MCF-7 cells. The cells were viewed under a Leica microscope for live imaging of ROS production using FITC filter at $\lambda_{\mathrm{ex}} 488 \mathrm{~nm}$ and $\lambda_{\mathrm{em}} 520 \mathrm{~nm}$.

\section{Results and discussion}

\subsection{Optical and morphological characterization}

IR 820 has been shown to be more stable than ICG which is a US FDA approved dye used for imaging and therapy. IR 820 has been encapsulated/conjugated with various biodegradable polymers such as chitosan and polyethylene glycol (PEG) amine for cancer theranostics [26,28].
We successfully encapsulated IR 820 dye with poloxamer and PCL blended polymeric nanoformulation. Most of the available drugs are hydrophobic in nature that can be easily incorporated in PCL. Poloxamer an amphiphilic surfactant has been extensively used in the delivery of low molecular weight drug and polypeptide [6,8,31].

Previous studies have shown that the addition of $10 \%$ poloxamer reduces the size of NPs and enhances the drug release from PCL matrix [8]. Hence, we chose for 90:10\% blend of PCL and poloxamer [6]. The formulation was further improved by the addition of GC in aqueous phase for the increase in cellular uptake efficiency. In addition, water soluble nature of GC does not affect the encapsulation of $\mathrm{pH}$ sensitive drugs. The schematic of NP synthesis has been shown in Fig. 1.

The NPs were prepared by double emulsion method. The hydrodynamic size of the NPs was found around $250 \pm 5 \mathrm{~nm}$ by DLS. Morphological analysis by FEG-SEM and TEM showed spherical shaped monodisperse NPs of size 100-220 nm (Figs. 2a and 3a). The surface charge was $+38 \pm 2 \mathrm{mV}$. The addition of poloxamer reduced the size of PP-IR nanoparticles from $336 \pm 5 \mathrm{~nm}$ to $250 \pm 5 \mathrm{~nm}$ as confirmed by DLS. This may be due to the hydrophobic interaction of polypropylene chain of poloxamer to PCL and hydrophilic interaction of polypropylene

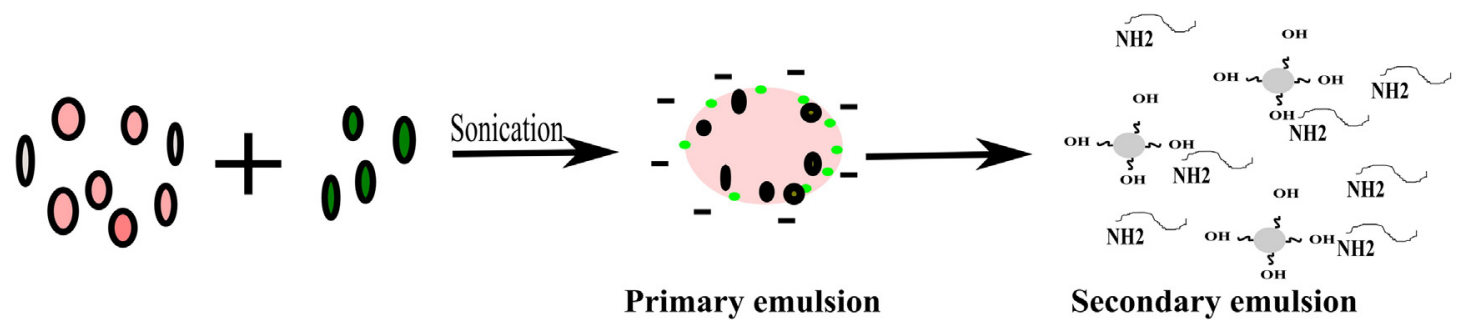

$\mathrm{O} \rightarrow$ PCL for drug encapsulation

$\mathbf{0} \rightarrow$ Poloxamer 188 as a apoptosis promoter

$0 \rightarrow$ IR820 as imaging and photothermal agent

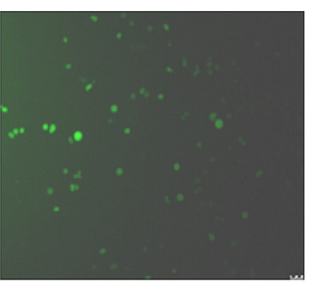

ROS production

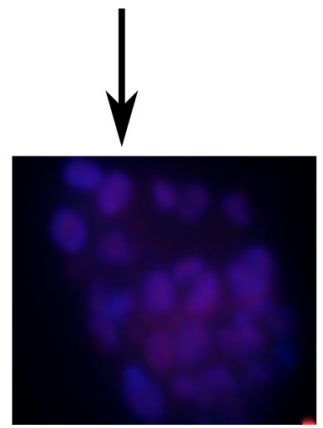

cell death after laser irradiation

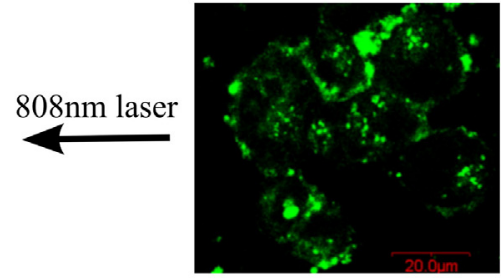

Cellular uptake

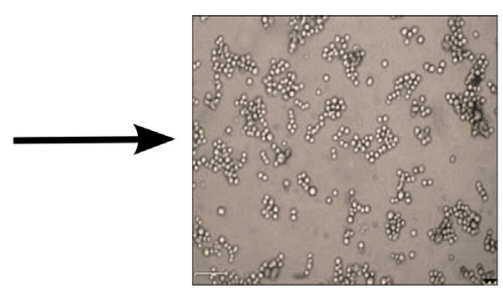

Cell death

(morpholology of cells

after $24 \mathrm{~h}$ )
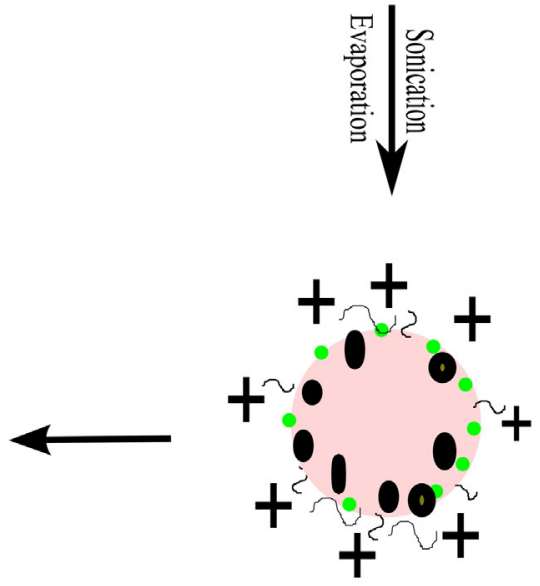

PVA used as a stabilizer

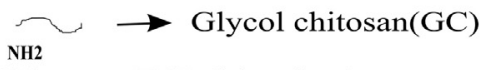

GC acts as TNF alpha stimulator

Fig. 1. Schematic showing the mechanism of combined laser immunotherapy of nanoformulation after 808 nm laser irradiation. 

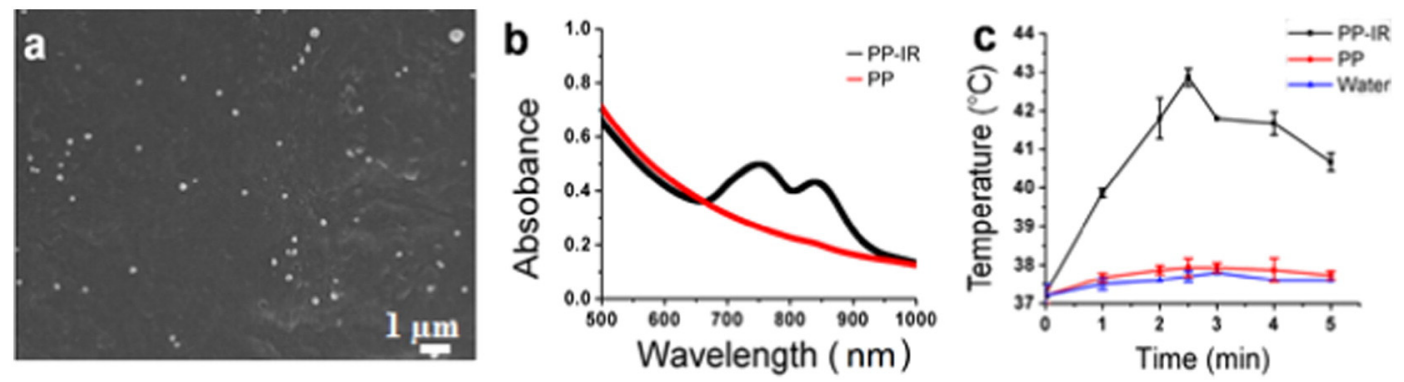

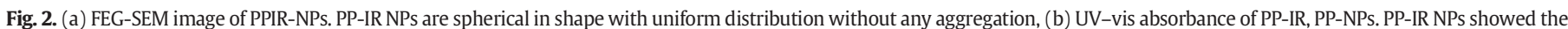

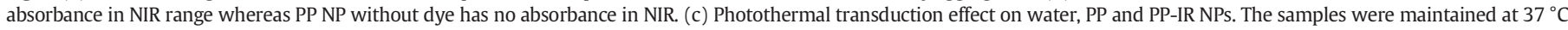
prior to laser application. A $0.5 \mathrm{~W} 808 \mathrm{~nm}$ laser was applied from 1 to 5 min time period. The rise in temperature was recorded with a NIR digital thermometer.

oxide chain with GC leading to compact nanoparticles. The size of NPs was similar to chitosan modified PLCL NPs prepared by Ranjan et al., wherein $5 \mathrm{mg} / \mathrm{mL}$ of chitosan was used to get a zeta potential of $+25 \mathrm{mV}$ [30]. We observed similar shape and size with $1 \mathrm{mg} / \mathrm{mL}$ of GC with a zeta potential $+38 \mathrm{mV}$. This gives the superiority of PP-IR nanoparticles compared to similar nanoformulation made with chitosan coating. The NP suspension was stable in water which may be attributed to high zeta potential due to GC coating on the surface of the nanoparticles. GC is a water-soluble polysaccharide yielding positive charge at pH 7.4. This results in maximum cellular uptake of NPs as cell surface has negative charge [13].

The UV-vis absorbance of PP-IR NPs showed two peaks around $750 \mathrm{~nm}$ and $845 \mathrm{~nm}$ respectively (Fig. 2b). The free dye has an absorbance at $820 \mathrm{~nm}$ in methanol. No peak was observed in NPs without dye (PP). The dye upon conjugation showed the shift in peak absorbance. Similar shift with IR dye was previously reported by Manchanda et al., wherein they have shown three peaks around 688, 702 and $836 \mathrm{~nm}$ upon interaction with PEG amine NPs [28]. In our case, peak shift remains in NIR windows, giving it an additional advantage for the photothermal application [29].

\subsection{Photothermal transduction}

The photothermal efficiency of NPs was determined by irradiating $\mathrm{NP}$ solutions by an $808 \mathrm{~nm}$ laser for $5 \mathrm{~min}$. Prior to laser treatment, samples were maintained at $37^{\circ} \mathrm{C}$. The temperature of NP suspension upon laser treatment was raised to $43^{\circ} \mathrm{C}$ in $2.5 \mathrm{~min}$ and subsequently temperature dipped till $5 \mathrm{~min}$. At the end of $5 \mathrm{~min}$, the temperature remained above $40^{\circ} \mathrm{C}$. However, the water and PP NPs did not show any significant increase in temperature upon laser treatment (Fig. 2c). The possible reason for the drop in the temperature after a 2.5 min laser treatment is poloxamer blending. Poloxamer has been shown to enhance the release of drug by forming pores in PCL matrix [8]. This leads to direct contact of the dye with the NIR laser beam that results in faster degradation of the dyes as observed in UV-vis absorbance of laser treated PP-IR NPs. Laser treated PP-IR NPs failed to show any absorbance inferring the complete degradation of dye inside it. The temperature around $43{ }^{\circ} \mathrm{C}$ has been shown to stimulate apoptosis in cancerous cells [32-34]. Apart from photothermal therapy, it can also be used for controlled drug delivery as there are no major morphological changes observed in TEM image in laser treated NPs (Fig. 3a-b).

\subsection{In vitro cytotoxicity and cellular uptake studies}

The biocompatibility test was conducted for $24 \mathrm{~h}$ on NIH3T3 cells in the concentration range from $100-1000 \mu \mathrm{g} / \mathrm{mL}$. The MTT results showed more than $80 \%$ cell viability even at a higher concentration of $1000 \mu \mathrm{g} / \mathrm{mL}$, suggesting its suitability for in vivo studies (Fig. 4a). The cell death at the higher concentration can be attributed to high positive charge and release of IR 820 dye by poloxamer. This can be minimized by adding ligands such as folic acid which impart the negative charge on the polymeric NP surface.

In vitro cellular uptake was studied by FITC-D loaded PP-IR NPs for $4 \mathrm{~h}$ in MCF-7 cells. PP-IR NPs were found in the cytoplasm as observed under a confocal laser scanning microscope (Fig. 4b). The high uptake of chitosan modified NPs has been reported due to the high affinity between positively charged NPs and negatively charged cancer cell membrane. The acidic environment of cancer cells enhances the protonation of chitosan modified NPs which leads to destabilization of cell membrane for increased cellular uptake [30].

The cells treated with PP-IR NPs and irradiated with an $808 \mathrm{~nm}$ laser for 5 min showed $80 \%$ cytotoxicity as compared to control cells (Fig. 4c). The cells treated either PP-NPs or laser alone failed to show any significant effect on cell viability. The high cytotoxicity of PP-IR NPs was attributed to the combined effect of IR 820 dye, GC, and poloxamer. IR 820 induced hyperthermia mediated cytotoxicity. The cytotoxic effect was further enhanced by GC and poloxamer. Earlier, poloxamer has been shown to enhance apoptosis in human chronic myelogenous leukemia cells [35] and reverse the multiple drug resistant cancers in combination with drugs $[8,9]$. In addition, chitosan has been reported to increase the

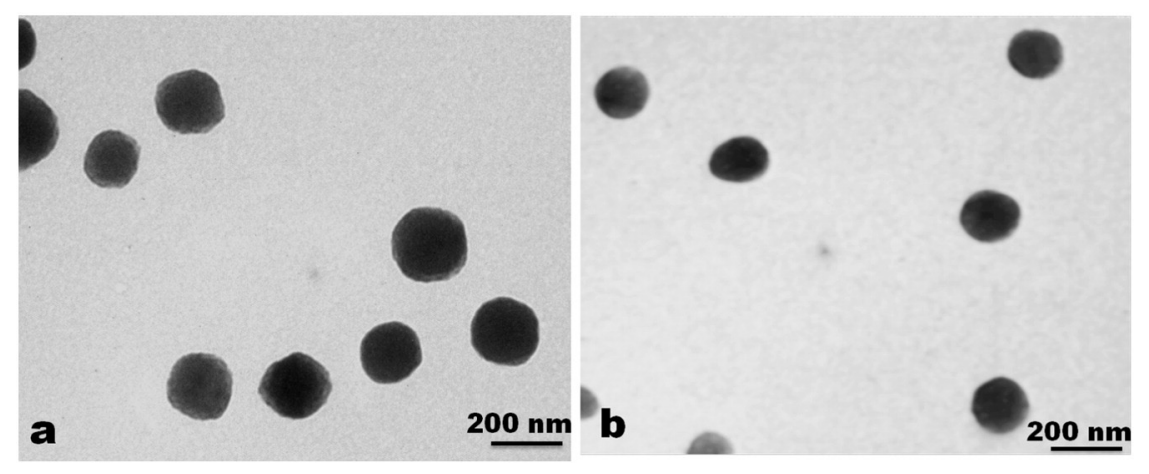

Fig. 3. (a) TEM image of PP-IR NPs before laser irradiation. (b) TEM image of PP-IR NPs after laser irradiation. 
a

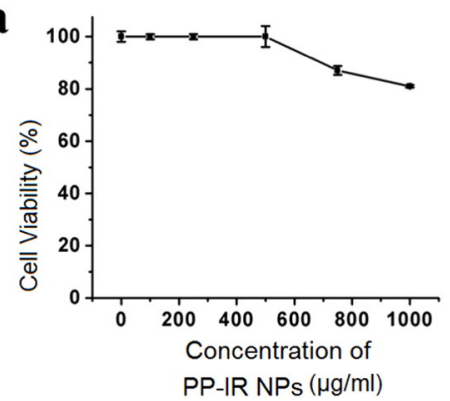

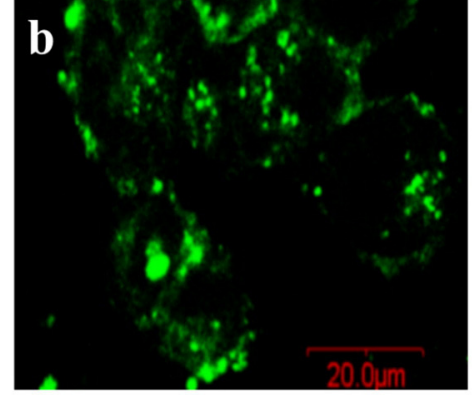

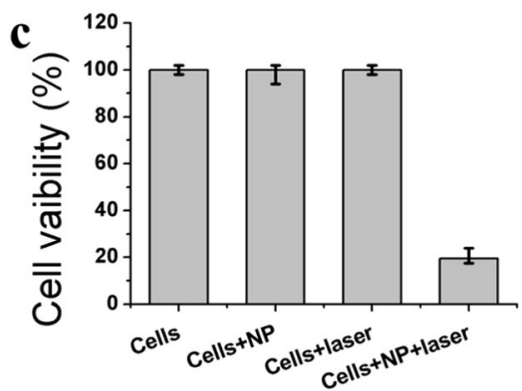

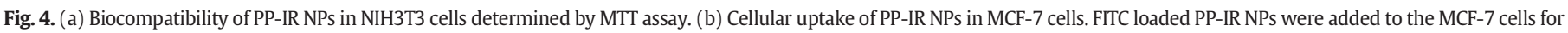

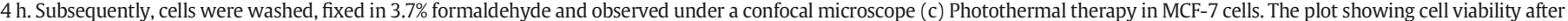
$24 \mathrm{~h}$ of PP-IR NP treatment.

TNF- $\alpha$ level even at low concentration. A three-fold increase in TNF- $\alpha$ activity was observed at the concentration of $90 \mu \mathrm{g} / \mathrm{mL}$ [36]. The increased level of TNF- $\alpha$ was shown to induce apoptosis and diminish the cell proliferation in MCF-7 cells [37].

To visualize the apoptosis and necrosis, live and dead/late apoptotic cells, cells were stained with Hoechst 33342 and PI respectively immediately after laser treatment (Fig. 5). The cells treated with laser and PPIR NPs showed significant cell death. Normal cells were stained faintly by Hoechst 33342 while apoptotic cells were stained intensively. PI did not diffuse into control cells, cells with PP-IR NPs and cells with laser treatment alone, as there were no membrane disruptions to get the dye enter. However, PI diffused in cells treated with NPs followed by laser irradiation showed fragmented nuclei. A faint stain of PI suggests the early stage of cell death. To study the ROS dependent cell death, we further checked the ROS level in all the groups. Elevated level of ROS has been observed in PP-IR NP treated cells irradiated by laser (Fig. 6a-d). The high level of ROS in PP-IR NP treated cells irradiated by laser was due to the combined effect of hyperthermia, poloxamer and stimulation of TNF- $\alpha$. High level of ROS leads to DNA fragmentation, leading to the formation of apoptotic bodies and ultimately cell death. Thus, we observed maximum apoptotic bodies in PP-IR NPs and lasertreated cells at the end of $24 \mathrm{~h}$ after PP-IR NP treatment (Fig. $6 \mathrm{e}-\mathrm{h}$ ).
This could help in cancer metastasis prevention as PP-IR NP treated cells irradiated by laser failed to attach to the substratum after $24 \mathrm{~h}$ of NP treatment (Fig. 6 e-h). The current study reflects the synergistic effect of hyperthermia, TNF- $\alpha$ and membrane destabilization by the poloxamer for effective treatment of drug-resistant estrogen positive breast cancer cells.

\section{Conclusions}

We have formulated a biocompatible and biodegradable, cost effective PP-IR NPs for bioimaging, photothermal therapy, and chemotherapy. The addition of poloxamer along with GC makes this nanosystem suitable for reversing multiple drug resistance. The addition of water soluble GC can also help in encapsulation of pH-sensitive drug and conjugation of specific targeting ligands such as folic acid. The combined effect of hyperthermia, chemotherapy and immunotherapeutics can also be used for the treatment of TNF- $\alpha$ resistant MCF-7 cells, apart from drug-resistant MCF-7 cells. A further in vivo study is needed to understand the efficacy of this system. This nanosystem can also be used for siRNA based delivery as poloxamer and GC have been shown to enhance the transfection efficiency. In the nutshell, this nanosystem could be promising a cheap and effective alternative to photoimmuno-chemotherapy in cancer research.
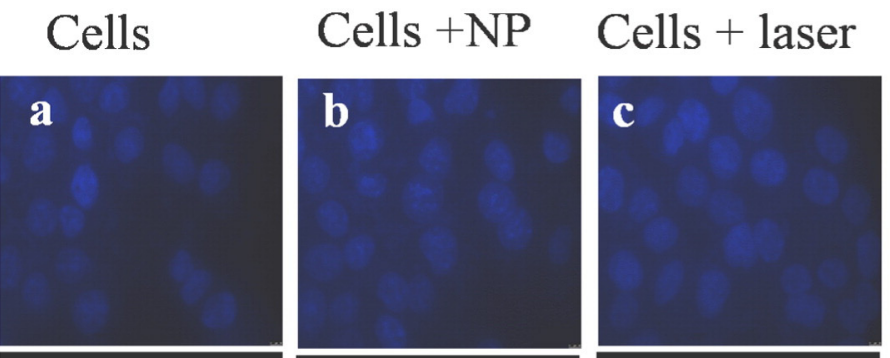

$$
\text { Cells + NP + laser }
$$
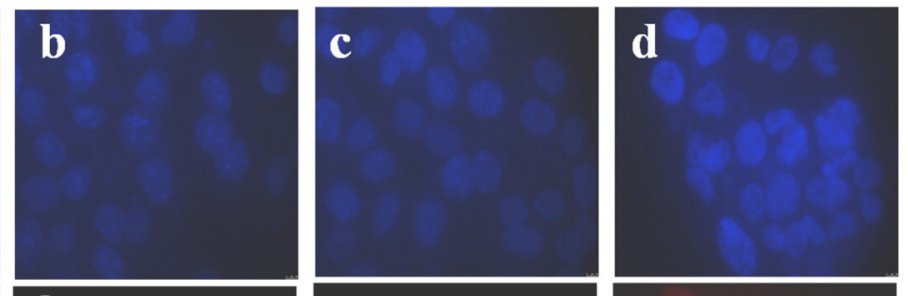

\section{Hoechst}
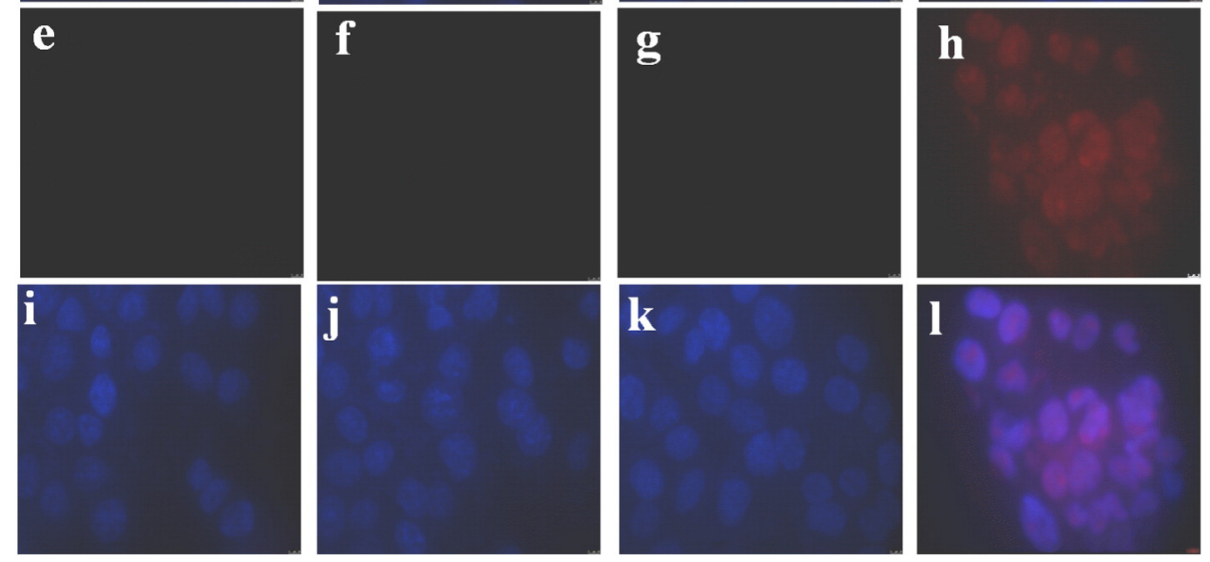

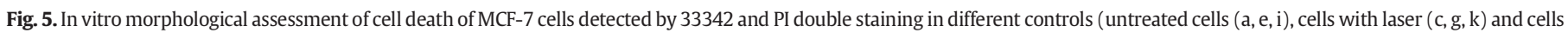
treated with bare NPs (b, f, g)) and cell treated with NPs and laser (d, h, i). 


\section{Cells}
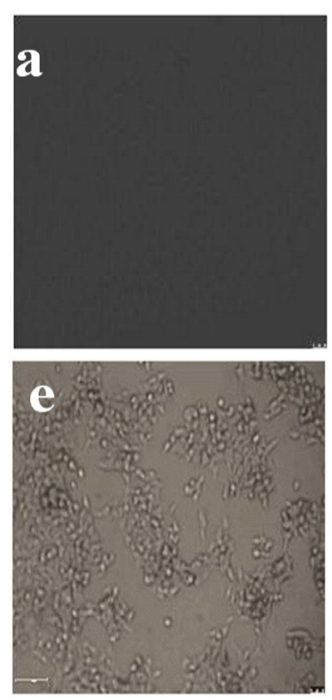

Cells +NP
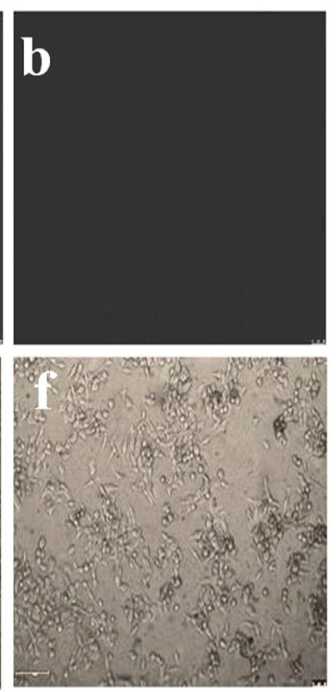

Cells + Laser
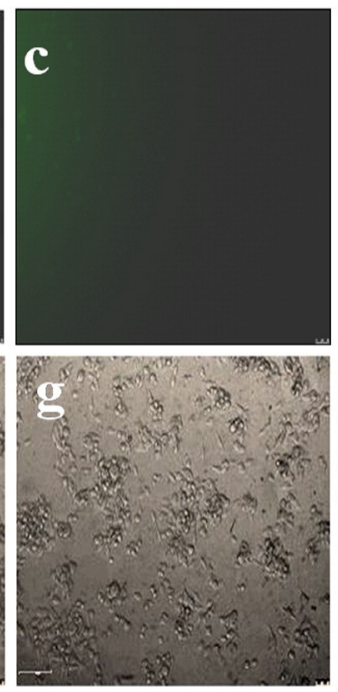

Cells + NP + Laser
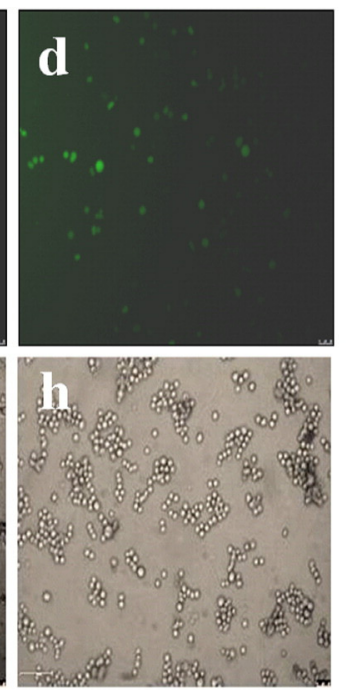

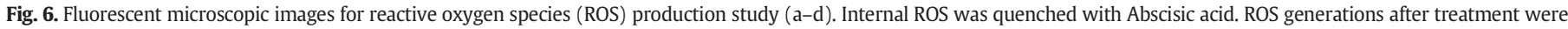

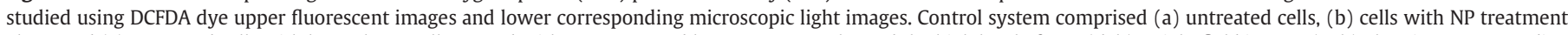

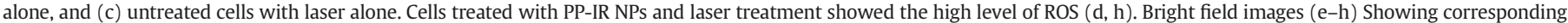
structural changes in cellular morphology after $24 \mathrm{~h}$.

\section{Acknowledgments}

The authors would like to acknowledge SAIF, CRNTS and Chemical Engineering Departments for characterization studies. We are thankful to Maruthi P. and Radhika P. for their help in manuscript writing and to Kunal K, Anadi G, S. Ray, Mukesh K., M. Thakur and A. Jha for their support during the experiment. P.K. is thankful to Indian Council of Medical Research, New Delhi for senior research fellowship [File no. 3/1/3/JRF2008/HRD-102(32238)]. The authors also acknowledge IITB-Healthcare (11IRSGHC001) for financial support.

\section{References}

[1] J.H. Adair, M.P. Parette, E.I. Altinoğlu, M. Kester, Nanoparticulate alternatives for drug delivery, ACS Nano 4 (2010) 4967-4970.

[2] A. Mahapatro, D.K. Singh, Biodegradable nanoparticles are excellent vehicle for site directed in-vivo delivery of drugs and vaccines, J. Nanobiotechnol. 9 (2011) 55.

[3] T.K. Dash, V.B. Konkimalla, Poly- $\varepsilon$-caprolactone based formulations for drug delivery and tissue engineering: a review, J. Control. Release 158 (2012) 15-33.

[4] A. Kumari, S.K. Yadav, S.C. Yadav, Biodegradable polymeric nanoparticles based drug delivery systems, Colloids Surf. B: Biointerfaces 75 (2010) 1-18.

[5] A.V. Kabanov, E.V. Batrakova, V. Yu, Pluronic block copolymers for overcoming drug resistance in cancer, 54 (2002) 759-779.

[6] F. Yan, C. Zhang, Y. Zheng, L. Mei, L. Tang, C. Song, H. Sun, L. Huang, The effect of poloxamer 188 on nanoparticle morphology, size, cancer cell uptake, and cytotoxicity, Nanomed. Nanotechnol. Biol. Med. 6 (2010) 170-178.

[7] K. Toth, R.B. Wenby, H.J. Meiselman, Inhibition of polymer-induced red blood cell aggregation by poloxamer 188, Biorheology 37 (2000) 301-312.

[8] L. Mei, Y. Zhang, Y. Zheng, G. Tian, C. Song, D. Yang, H. Chen, H. Sun, Y. Tian, K. Liu, Z. Li, L. Huang, A novel docetaxel-loaded poly ( $\varepsilon$-caprolactone)/pluronic F68 nanoparticle overcoming multidrug resistance for breast cancer treatment, Nanoscale Res. Lett. 4 (2009) 1530-1539.

[9] P.N. Gupta, S. Jain, C. Nehate, N. Alam, V. Khare, R.D. Dubey, A. Saneja, S. Kour, S.K. Singh, Development and evaluation of paclitaxel loaded PLGA:poloxamer blend nanoparticles for cancer chemotherapy, Int. J. Biol. Macromol. 69C (2014) 393-399.

[10] P. Caaman, A. Sa, F. Domı, PLGA:poloxamer and PLGA:poloxamine blend nanoparticles: new carriers for gene delivery, Society (2005) 271-278.

[11] I. Tsoneva, I. Iordanov, A.J. Berger, T. Tomov, B. Nikolova, N. Mudrov, M.R. Berger, Electrodelivery of drugs into cancer cells in the presence of poloxamer 188, J. Biomed. Biotechnol. 2010 (2010).

[12] H. Koo, K.H. Min, S.C. Lee, J.H. Park, K. Park, S.Y. Jeong, K. Choi, I.C. Kwon, K. Kim, Enhanced drug-loading and therapeutic efficacy of hydrotropic oligomer-conjugated glycol chitosan nanoparticles for tumor-targeted paclitaxel delivery, J. Control. Release 172 (2013) 823-831.

[13] G. Bajaj, G. William, V. Alstine, Y. Yeo, Zwitterionic chitosan derivative, a new biocompatible pharmaceutical excipient, prevents endotoxin-mediated cytokine release, 7 (2012) e30899.
[14] S. Kim, D.J. Lee, D.S. Kwag, U.Y. Lee, Y.S. Youn, E.S. Lee, Acid pH-activated glycol chitosan/fullerene nanogels for efficient tumor therapy, Carbohydr. Polym. 101 (2014) 692-698.

[15] R.N. Mitra, Z. Han, M. Merwin, M. Al Taai, S.M. Conley, M.I. Naash, Synthesis and characterization of glycol chitosan DNA nanoparticles for retinal gene delivery, ChemMedChem 9 (2014) 189-196.

[16] J. Young, S. Song, S. Jin, S. Park, K. Kim, M. Goo, S. Son, H. Koo, I. Chan, J. Hoon, S. Young, S. Hwa, K. Kim, Cancer-targeted MDR-1 siRNA delivery using self-crosslinked glycol chitosan nanoparticles to overcome drug resistance, J. Control. Release 198 (2015) 1-9.

[17] S. Lee, S.W. Kang, J.H. Ryu, J.H. Na, D.E. Lee, S.J. Han, C.M. Kang, Y.S. Choe, K.C. Lee, J.F Leary, K. Choi, K.H. Lee, K. Kim, Tumor-homing glycol chitosan-based optical/PET dual imaging nanoprobe for cancer diagnosis, Bioconjug. Chem. 25 (2014) 601-610.

[18] J. Rhee, O.K. Park, A. Lee, D.H. Yang, K. Park, Glycol Chitosan-based Fluorescent Theranostic Nanoagents for Cancer Therapy, 2014 6038-6057.

[19] C.S., R., J. Kumar, V., R., M., V., A. Abraham, Laser immunotherapy with gold nanorods causes selective killing of tumour cells, Pharmacol. Res. 65 (2012) 261-269.

[20] P. Zhao, M. Zheng, C. Yue, Z. Luo, P. Gong, G. Gao, Z. Sheng, C. Zheng, L. Cai, Improving drug accumulation and photothermal efficacy in tumor depending on size of ICG loaded lipid-polymer nanoparticles, Biomaterials 35 (2014) 6037-6046.

[21] M. Zheng, C. Yue, Y. Ma, P. Gong, P. Zhao, C. Zheng, Z. Sheng, P. Zhang, Z. Wang, L. Cai, Single-step assembly of DOX/ICG loaded lipid-polymer nanoparticles for highly effective chemo-photothermal combination therapy, ACS Nano 7 (2013) 2056-2067.

[22] M. Zheng, P. Zhao, Z. Luo, P. Gong, C. Zheng, P. Zhang, C. Yue, D. Gao, Y. Ma, L. Cai, Robust ICG theranostic nanoparticles for folate targeted cancer imaging and highly effective photothermal therapy, ACS Appl. Mater. Interfaces 6 (2014) 6709-6716.

[23] W. Guo, L. Zhang, J. Ji, W. Gao, J. Liu, M. Tong, Breast cancer sentinel lymph node mapping using near-infrared guided indocyanine green in comparison with blue dye, Tumour Biol. 35 (4) (2014), http://dx.doi.org/10.1007/s13277-013-1399-2.

[24] V. Melikian, J.D. Eddy, A. Paton, The stimulant effect of drug on indocyanine green clearance by liver, Gut 13 (1972) 755.

[25] A. Fernandez-Fernandez, R. Manchanda, T. Lei, D.A. Carvajal, Y. Tang, S.Z.R. Kazmi, A.J. McGoron, Comparative study of the optical and heat generation properties of IR820 and indocyanine green, Mol. Imaging 11 (2012) 99-113.

[26] S. Srinivasan, R. Manchanda, A. Fernandez-Fernandez, T. Lei, A.J. Mcgoron, Nearinfrared fluorescing IR820-chitosan conjugate for multifunctional cancer theranostic applications, J. Photochem. Photobiol. B Biol. 119 (2013) 52-59.

[27] P. Huang, P. Rong, A. Jin, X. Yan, M.G. Zhang, J. Lin, H. Hu, Z. Wang, X. Yue, W. Li, G Niu, W. Zeng, W. Wang, K. Zhou, X. Chen, Dye-loaded ferritin nanocages for multimodal imaging and photothermal therapy, Adv. Mater. (2014) 6401-6408.

[28] A. Fernandez, R. Manchanda, T. Lei, Covalent IR820-PEG-diamine nanoconjugates for theranostic applications in cancer, Int. J. Nanomedicine (2014) 4631-4648.

[29] P. Kumar, R. Srivastava, IR 820 stabilized multifunctional polycaprolactone glycol chitosan composite nanoparticles for cancer therapy, RSC Adv. 5 (2015) 56162 http://dx.doi.org/10.1039/C5RA05997F.

[30] A.P. Ranjan, K. Zeglam, A. Mukerjee, S. Thamake, J.K. Vishwanatha, A sustained release formulation of chitosan modified PLCL:poloxamer blend nanoparticles loaded with optical agent for animal imaging, Nanotechnology 22 (2011) 295104.

[31] L. Minai, D. Yeheskely-Hayon, D. Yelin, High levels of reactive oxygen species in gold nanoparticle-targeted cancer cells following femtosecond pulse irradiation, Sci. Rep. 3 (2013) 2146. 
[32] K. Fuller, R. Issels, D. Slosman, Eur. J. Cancer 30 (1994) 1884-1891.

[33] B.V. Harmon, Y.S. Takano, C.M. Winterford, G.C. Gobé, Int. J. Radiat. Biol. 59 (1991) 489-501.

[34] D.K. Chatterjee, P. Diagaradjane, S. Krishnan, Ther. Deliv. 2 (2011) 1001-1014.

[35] S.M. Moghimi, A.C. Hunter, C.M. Dadswell, S. Savay, C.R. Alving, J. Szebeni, Causative factors behind poloxamer 188 (Pluronic F68, Flocor)-induced complement activation in human sera. a protective role against poloxamer-mediated complement activation by elevated serum lipoprotein levels, Biochim. Biophys. Acta 1689 (2004) 103-113.
[36] R. Chen, X. Wang, X. Yao, X. Zheng, J. Wang, X. Jiang, Near-IR-triggered photothermal/ photodynamic dual-modality therapy system via chitosan hybrid nanospheres, Biomaterials 34 (2013) 8314-8322.

[37] M.E. Burow, C.B. Weldon, Y. Tang, G.L. Navar, S. Krajewski, J.C. Reed, T.G. Hammond, S. Clejan, B.S. Beckman, Differences in susceptibility to tumor necrosis factor alphainduced apoptosis among MCF-7 breast cancer cell variants, Cancer Res. 58 (1998) 4940-4946. 